\title{
Advantage of a predictive control law for extreme adaptive optics imaging
}

\author{
B. Le Roux ${ }^{1}$ and M. Carbillet ${ }^{2}$ \\ ${ }^{1}$ INAF-Osservatorio Astrofisico di Arcetri, Largo E. Fermi, I-50125 Firenze \\ email: leroux@arcetri.astro.it \\ ${ }^{2}$ LUAN-UMR 6525, Université de Nice-Sophia Antipolis, Parc Valrose, F-06108 Nice cedex 2 \\ email: marcel.carbillet@unice.fr
}

\begin{abstract}
We study the application of a predictive control law based on a Kalman filter for an extreme adaptive optics system. In particular, we discuss the minimization of temporal error and show the evolution of prediction errors with the order of the model. We also discuss the choice of the optimal temporal frequency as a function of the control law and the level of noise. Finally, the gain expected with respect to a non-predictive law is presented.
\end{abstract}

Keywords. atmospheric effects; instrumentation: adaptive optics; stars: imaging.

\section{Introduction}

Imaging faint astrophysical objects near bright stars, like exoplanets, is a real challenge for modern astrophysical instrumentation. Recently, a planet has been imaged by Chauvin et al. (2005), using the NACO instrument: the faint companion was one hundred times fainter than the brown dwarf. In this case the atmospherical correction provided by the adaptive optics [OA] system NAOS was enough to image the planet, but in general an instrument dedicated to exoplanets imaging should be able to image objects $10^{5}$ to $10^{10}$ times fainter than the star. This means, among others, that a very powerfull AO system is needed. This is called an eXtreme AO [XAO] system, characterized by a resulting Strehl ratio in the imaging band as extreme as $90-95 \%$. This challenge has recently motivated the publication of a number of ideas to improve $\mathrm{AO}$, for example in wave-front sensing [WFS] (Nicolle et al. (2004), Vérinaud et al. (2005)).

We propose here to improve again the AO performance by considering a predictive control law, applying the Kalman-filter-based optimal control law (Le Roux et al. (2004)) to the XAO case. We first remind the principle of this optimal approach in Sec. 2. In Sec. 3 we then discuss some of its limits and the way to apply it to XAO, showing how using a high order autoregressive $[\mathrm{AR}]$ model to describe the turbulence evolution would decrease the temporal error. We also discuss the trade-off to be found between the temporal error and the WFS error due to the noise, leading to the notion of optimal temporal frequency. Finally we show in Sec. 4 how decreasing the temporal frequency and using a predictive control law permit to increase the performance of an XAO system, with respect to simply use an integrator control law with a high temporal frequency.

\section{State-space formalism and optimal control for AO}

The optimal control law used here (Le Roux et al. (2004)) is based on a Kalman filter in a state-space formalism, built on a state-space model recalled hereafter.

Let us first consider the chronology of events in an AO system: CCD integration for WFS, read-out of CCD, computation and application of new voltages for the deformable 
mirror. Assuming the same time $T$ for the CCD integration and for the global delay, there is a $2 T$ time-lag between the measurement and the application of the correction, time-lag to which is due the so-called temporal error.

The Kalman filter is an estimator of the turbulent phase that is based on a physical representation of the system turbulence $\oplus \mathrm{AO}$, the so-called state model. This model should sum up the behaviour of the system and the chronology described before which can be read in three fundamental equations:

- the definition of the residual phase: $\phi^{\text {res }}=\phi^{\text {tur }}-\phi^{\text {cor }}=\phi^{\text {tur }}-\mathbf{N u}$, where $\mathbf{N}$ is the matrix that links the voltages basis to the turbulent modes basis, $\mathbf{u}$ are the voltages, $\phi^{\text {tur }}, \phi^{\text {res }}$ and $\phi^{\text {cor }}$ are denoting the turbulent, residual and correction phase;

- the definition of the measurement: $\mathbf{Y}_{n}=\mathcal{D} \phi_{n-1}^{\text {res }}+\mathbf{w}_{n}$, where $\mathcal{D}$ is the interaction matrix, and $\mathbf{w}_{n}$ the measurement noise at instant $n T$;

- the equation of evolution of the turbulence written as: $\phi_{n+1}^{\mathrm{tur}}=\mathcal{F}\left[\phi_{n}^{\mathrm{tur}}, \phi_{n-1}^{\mathrm{tur}}, \ldots, \phi_{n-p+1}^{\mathrm{tur}}\right]+$ $\nu_{n}$, where $\nu$ is a white noise and $\mathcal{F}$ is being choosen as an AR model of order $p\left(\mathrm{AR}_{p}\right)$.

In Le Roux et al. (2004), and for the sake of simplicity, the evolution of the turbulence was described by the $\mathrm{AR}_{1}$ model: $\phi_{n+1}^{\text {tur }}=\mathbf{A} \phi_{n}^{\text {tur }}+\nu_{n}$. It is then possible to describe the system by choosing the state vector: $\mathbf{X}_{n}=\left(\begin{array}{lllll}\phi_{n+1}^{\text {tur }} & \phi_{n}^{\text {tur }} & \phi_{n-1}^{\text {tur }} & \mathbf{u}_{n-1} & \mathbf{u}_{n-2}\end{array}\right)^{T}$. The so-called state-space model is then:

$$
\begin{aligned}
\mathbf{X}_{n+1}= & \left(\begin{array}{lllll}
\mathbf{A} & 0 & 0 & 0 & 0 \\
\mathbf{I d} & 0 & 0 & 0 & 0 \\
0 & \mathbf{I d} & 0 & 0 & 0 \\
0 & 0 & 0 & 0 & 0 \\
0 & 0 & 0 & \mathbf{I d} & 0
\end{array}\right) \mathbf{X}_{n}+\left(\begin{array}{l}
0 \\
0 \\
0 \\
\mathbf{I d} \\
0
\end{array}\right) \mathbf{u}_{n}+\left(\begin{array}{l}
\nu_{n} \\
0 \\
0 \\
0 \\
0
\end{array}\right) \\
\mathbf{Y}_{n}= & \mathcal{D}\left(\begin{array}{lllll}
0 & 0 & \mathbf{I d} & 0 & -\mathbf{N}
\end{array}\right) \mathbf{X}_{n}+\mathbf{w}_{n} .
\end{aligned}
$$

The enhancement brought by the Kalman approach with respect to a more classical approach, the Optimized Modal Gain Integrator [OMGI] in classical AO (Gendron \& Léna (1994)), was presented in Le Roux et al. (2004), where an improvement essentially due in that case to the predictive ability of the optimal control law was obtained. The use of a temporal modeling of the turbulence allows to predict its evolution during the $2 T$ delay between WFS and correction and then reduces the temporal delay. The $\mathrm{AR}_{1}$ model used to characterize the temporal evolution of the atmospherical turbulence was enough to improve the performance of generic AO and MCAO systems.

But the temporal decorrelation of such a model is exponential while the turbulence one is slower. Figure 1 presents the behaviour of the temporal decorrelation of a TaylorKolmogorov turbulence and two AR1 models. Increasing the order of the model would allow to better model the temporal evolution of the turbulence and therefore to better predict its behavior.

\section{Control for XAO: optimal prediction and noise filtering}

\subsection{Prediction and noise filtering}

Current projects of XAO systems usually consider high sampling frequencies, higher than $1 \mathrm{kHz}$, and WFS able to sense very high spatial frequencies, as a Shack-Hartmann [SH] with many sub-apertures (e.g. $40 \times 40$ for an $8-\mathrm{m}$ telescope). The first consequence of such a configuration is a very low signal for each sub-aperture (i.e. a high photon noise). This should be considered as a consequence of the use of an integrator control law. 


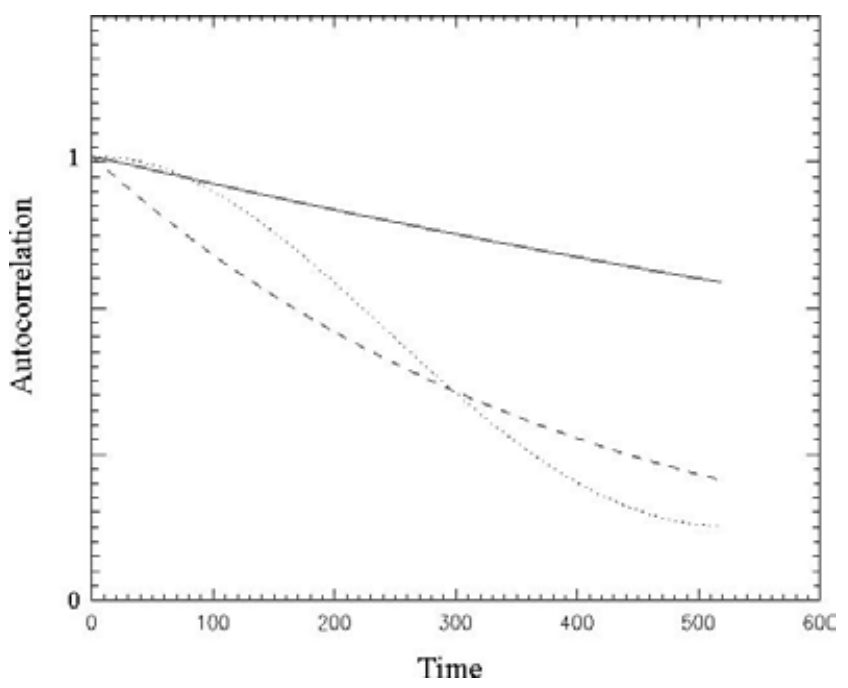

Figure 1. General form of the temporal decorrelation for the atmospheric turbulence [dotted line] and for two AR1 models [continuous line and dashed line].

The choice of the temporal frequency $f_{\text {temp }}$ being a trade-off between decreasing the temporal error by increasing $f_{\text {temp }}$ and decreasing the photon noise by decreasing $f_{\text {temp }}$, there is an optimal value of $f_{\text {temp }}$ to be found, minimizing the sum of the two error terms. In the case of a non-predictive control law, as the integrator one, the only way to decrease the temporal error is to highly increase $f_{\text {temp. }}$. In the other hand a predictive control law allows to decrease the temporal error by using an efficient temporal model to predict the turbulent evolution. The resulting optimal $f_{\text {temp }}$ is then lower, allowing to simultaneously reduce the temporal error, thanks to prediction, and the photon noise.

We propose here to compare the performance of a system based on an integrator control law with a high $f_{\text {temp }}$ and a system based on a predictive control law, here based on a Kalman filter, with a lower $f_{\text {temp }}$.

There are many questions to be answered. First of all, the order of the AR model necessary to do such a prediction is a crucial parameter that decides its practical feasibility. It has to be determined, as well as $f_{\text {temp }}$ in the predictive case, for a given photon noise. Finally, we should express the gain brought by this predictive approach.

\subsection{Choice of the state-space model for XAO}

The choice of the state-space model for XAO depends essentially upon the choice of the order of the AR model used. In order to address the influence of the AR model order on the prediction error we have simulated a turbulence made up of 7 layers moving in random angles with a wind speed of $12 \mathrm{~m} / \mathrm{s}$ and following a Kolmogorov spectrum with a Fried parameter of $12.5 \mathrm{~cm}$ (at $500 \mathrm{~nm}$ ), over an $8-\mathrm{m}$ telescope. The turbulent phase arriving on the telescope aperture is decomposed onto the first 30 radial orders of the Zernike modes basis for 5000 iterations, at a rate of $500 \mathrm{~Hz}$ (the temporal frequency has been choosen here as an example).

For each Zernike mode, we fit an $\mathrm{AR}_{1}, \mathrm{AR}_{2}, \ldots, \mathrm{AR}_{11}$ on 500 iterations. We then use these AR models to predict the evolution of the turbulence in the next 500 iterations. The computed resulting global prediction error is plotted on Fig. 2: we obtain that it decreases continously with the order of the $\mathrm{AR}$ model. Note that from an $\mathrm{AR}_{1}$ to an $\mathrm{AR}_{11}$ model a huge gain in variance is obtained, but this gain is almost already acheived with a $4^{\text {th }}$ order model, which then represents the best order in this case.

Now let us remark that in reality, the optimal order of the model depends upon $f_{\text {temp }}$. The ability of the model to represent the turbulence evolution and hence to predict it 


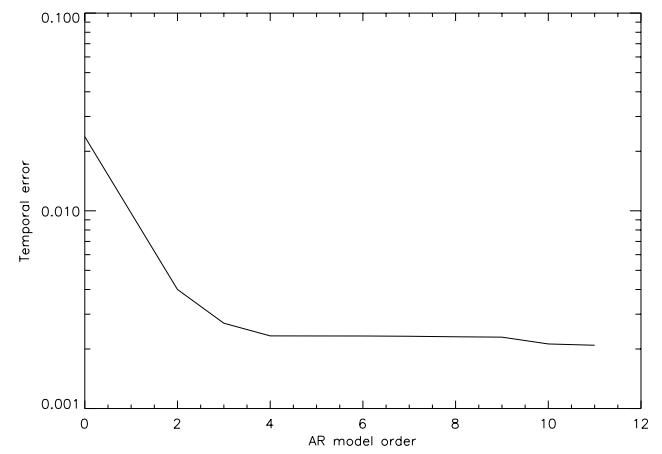

Figure 2. AR model prediction error vs. order (order 0 means no prediction).

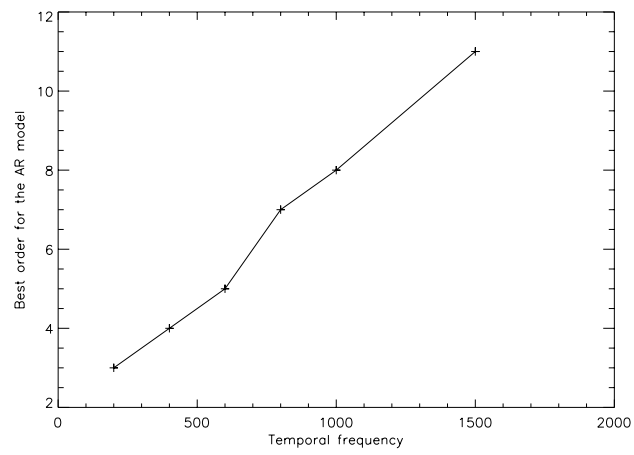

Figure 3. Best order of the AR model vs. temporal frequency.

can be linked to the temporal range on which it is written, meaning the order of the model times the temporal frequency. As an example, 4 iterations at $500 \mathrm{~Hz}$ correspond to the same temporal range than 8 iterations at $1.5 \mathrm{kHz}$. Of course this argument is totally qualitative and needs to be quantified.

We reproduced the same steps as before with the same parameters but for a range of $f_{\text {temp }}$ between $100 \mathrm{~Hz}$ and $1 \mathrm{kHz}$, in order to find the best order for the AR model. The best $f_{\text {temp }}$ is the frequency from which there is no relevant gain in increasing again the frequency. Figure 3 presents the best model order $p_{\text {best }}$ vs. $f_{\text {samp. }}$. The expected behaviour of $p_{\text {best }}$ increasing with $f_{\text {ech }}$ is clearly shown.

\subsection{Choice of the temporal frequency and gain obtained}

In a second step, it is possible to quantify the improvement brought by a predictive approach with respect to the integrator control law and to determine the corresponding $f_{\text {temp. }}$. We have hence simulated again the same Kolmogorov turbulence. The temporal frequency explored a range from 100 to $2000 \mathrm{~Hz}$, and for each frequency we computed the same way as before the parameters of the $\mathrm{AR}_{p}$ model, with $p$ optimized, as in Fig. 3 .

Such a control law is based on the same type of model that the one presented in Sec.sec:rappels but with more turbulent phase vectors in the state vector, in order to be able to write the full $\mathrm{AR}$ model. To use an $\mathrm{AR}_{5}$ model, the state vector must be $\mathbf{X}_{n}=\left(\begin{array}{lllllll}\phi_{n+1}^{\text {tur }} & \phi_{n}^{\text {tur }} & \phi_{n-1}^{\text {tur }} & \phi_{n-2}^{\text {tur }} & \phi_{n-3}^{\text {tur }} & \mathbf{u}_{n-1} & \mathbf{u}_{n-2}\end{array}\right)^{T}$, in order to be able to write $\phi_{n+2}^{\text {tur }}$ (within $\mathbf{X}_{n+1}$ ) as a function of $\phi_{n+1}^{\text {tur }}, \phi_{n}^{\text {tur }}, \phi_{n-1}^{\text {tur }}, \phi_{n-2}^{\text {tur }}$ and $\phi_{n-3}^{\text {tur }}$ (within $\mathbf{X}_{n}$ ). Of course this means that all vectors and matrices are much bigger than with an $\mathrm{AR}_{1}$ model. If we consider in the same time that the number of measurements (given for example by a $40 \times 40 \mathrm{SH}$ ) can be already large and that recent propositions to increase the sensitivity of WFS in XAO (Le Roux et al. (2005)) could increase even more this number, one should understand that an XAO system represents a huge number of parameters to deal with. A full study must be done to understand how the computing time can be decreased, probably by using sparse matrix properties.

\section{Discussion}

Figure 4 shows, as a function of the temporal frequency, the temporal errors corresponding to the predictive case [full line] and the non-predictive case [dashed line]. The photon noise as a function of the temporal frequency is also plotted [dotted line], as well as the sum of the temporal errors and of the noise [dashed-dotted and dashed-triple-dotted 


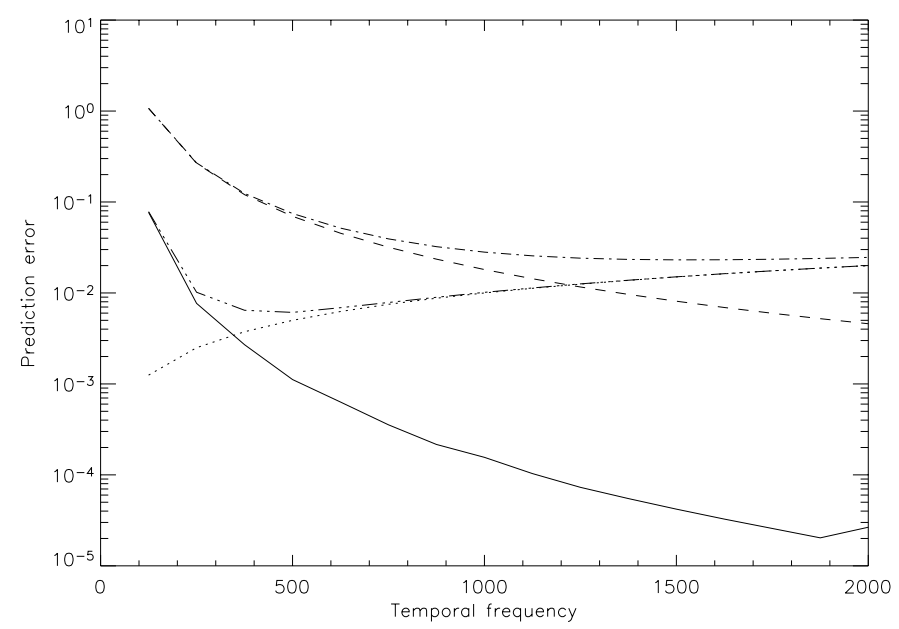

Figure 4. Temporal errors in the integrator case and the predictive control case, and WFS error due to photon noise.

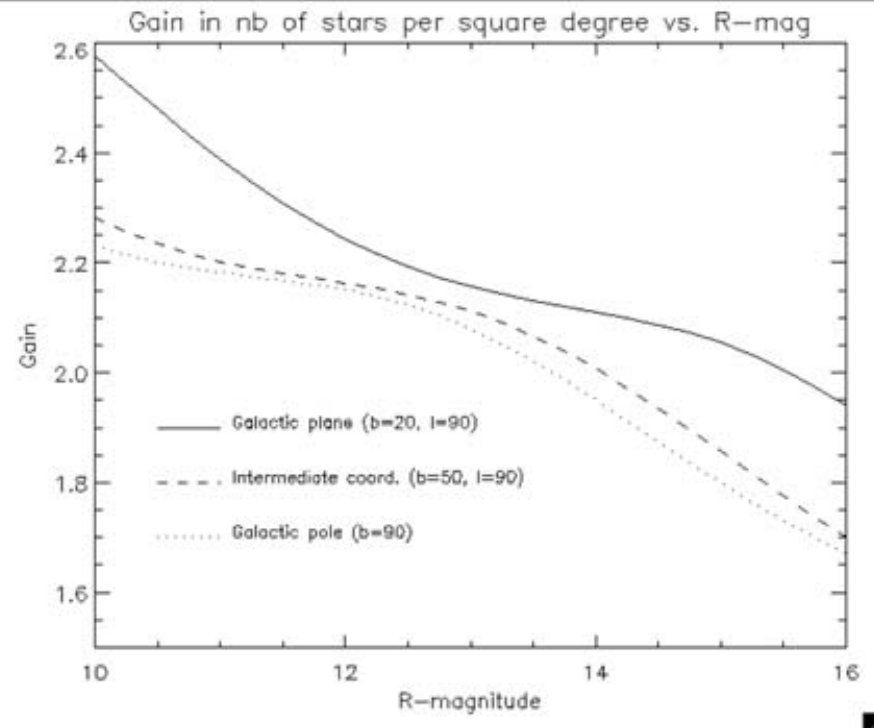

Figure 5. Evolution of the number of candidate stars for a predictive approach with respect to a non-predictive approach.

lines] in order to be able to compare the relevant parameters, which are the sum of both error terms. In the predictive case, an optimum is obtained at a temporal frequency $f_{\text {pred }}$ which is about half the optimal frequency obtained in the non-predictive case. In terms of variance of error, choosing $f_{\text {pred }}$ and a predictive control reduces the variance by a factor 2 .

It should be indeed noticed that the goal of this first study is the understanding of the prediction aspect of the Kalman-based filter. It does not take into account parameters like for example a difference of noise propagation between predictive and non-predictive control. A full AO simulation would be necessary to confirm the gain of the predictive approach in a realistic case. We are currently implementing an optimal control based on a high-order AR-model within the Software Package CAOS (Carbillet et al. (2005)) in order to simulate a full $\mathrm{AO}$ system working with a control law and quantitatively compare it to an integrator control law.

For what concerns the number of candidate stars for exoplanet search, the previous result has the following implication. A gain of two in variance of residual phase implies a gain of one mag. in limiting magnitude. Figure 5 shows the resulting gain in number 
of observable stars by square degree vs. the magnitude and for three typical positions in the sky, based on the Bachall \& Soneira model of distribution of stars (Bahcall \& Soneira (1984)). This gain in number of observable stars is in average in the field larger than 2 .

\section{References}

Chauvin, G., Lagrange, A.-M., Dumas, C., et al., 2005, Astron. Astrophys., 438 (2), L25

Nicolle, M., Fusco, T., Rousset, G., \& Michau, V., 2004, Opt. Letters, 29 (23), 2743

Vérinaud, C., Le Louarn, M., Korkiakoski, V., \& Carbillet, M., 2005, MNRAS, 357 (1), L26

Le Roux, B., Conan, J.-M., Kulcsár, C., et al., 2004, J. Opt. Soc. Am. A, 21(7), 1261

Gendron, É. \& Léna, P., 1994, Astron. Astrophys., 291 (1), 337

Carbillet, M., Vérinaud, C., Femenía, B., et al., 2005, MNRAS, 356 (4), 1263

Le Roux, B., Coyne, J., \& Ragazzoni, R., 2005, App. Opt., 44 (2), 171

Bahcall, J. N. \& Soneira, R. M., 1984, Ap. J. Suppl. Ser., 55, 67 